\title{
The effectiveness of the Raja Isteri Pengiran Anak Saleha Appendicitis Score in the diagnosis of acute appendicitis in elderly patients
}

\author{
Osman Bardakci ${ }^{1, *}$, Faik Tatli ${ }^{1}$
}

${ }^{1}$ Harran University, Faculty of Medicine, Department of General Surgery, Şanlıurfa, Turkey

\section{*Correspondence}

drosmanbardakci@gmail.com (Osman Bardakci)

\begin{abstract}
Acute appendicitis (AA) is the most common cause of acute abdominal surgeries, accounting for $15 \%$ of all emergency visits due to acute abdominal pain in patients aged over 60 years. Appendicitis is reported as the third most common cause of abdominal surgery in geriatric patients. In this current retrospective study, the records of 68 patients aged over 65 years, who were treated by surgical resection in our clinic diagnosed for AA between February 2015 and February 2020, were analyzed. The age, gender, duration of hospital stay of the patients and, histopathological results of appendectomy specimens were recorded. The Raja Isteri Pengrian Anak Saleha Appendicitis (RIPASA) Score of the patients was calculated according to the clinical history, physical examination, and laboratory parameters of the patients. According to the RIPASA scores, the patients were divided into three groups as the low-score group (4 to 7), the intermediate-score group (7.5 to 11.5), and the high-score group $(\geq 12)$. The groups were compared on the basis of the microscopic diagnosis. According to the calculated RIPASA score, there were 12 patients in the low-score group, 44 patients in the intermediate-score group, and 12 patients in the high-score group. When the results were classified based on the pathology, we detected non-complicated conditions such as a normal appendix or reactive lymphoid hyperplasia in ten patients and catarrhal stage appendicitis in 38 patients along with complicated (gangrenous or perforated) appendicitis in 20 patients. In conclusion, the RIPASA score can be used in elderly patients considering its high accuracy rate. In elderly patients with a high RIPASA score, the pathological stage of the appendectomy specimen may also be complicated (perforated or gangrenous) appendicitis. Therefore, the mortality and morbidity rates may increase with increased complication rates.
\end{abstract}

\section{Keywords}

Acute appendicitis; Elderly patients; Raja Isteri Pengiran Anak Saleha Appendicitis Score

\section{Introduction}

Acute appendicitis (AA) is the most common cause of acute abdominal surgery [1]. The incidence of acute appendicitis is approximately $233: 100000$ that varies from $7 \%$ to $10 \%$ throughout life [2]. Furthermore, AA accounts for $15 \%$ of all emergency visits due to acute abdominal pain in patients aged over 60 years and is the third most common cause of abdominal surgery in geriatric patients $[3,4]$.

Acute appendicitis is mainly diagnosed by anamnesis, physical examination, and laboratory parameters. Notwithstanding, the diagnosis of AA may be delayed due to the difficulties of examination of children, elderly patients, and pregnant women. Delayed diagnosis leads to increased morbidity and mortality rates in these populations. Ultrasonography, tomography, and a series of scoring systems are also used to support the diagnosis. Scoring systems are diagnostic tools that are used to clearly differentiate between acute appendicitis and nonspecific abdominal pain [5]. The Raja Isteri Pengiran Anak Saleha Appendicitis (RIPASA) Scoring System is a widely used rapid diagnostic tool since $2010[6,7]$ (Table 1). The RIPASA score has a sensitivity and specificity of $98 \%$ and $83 \%$, respectively, in diagnosing acute appendicitis [8].

An early diagnosis of AA is particularly important in elderly people. In a study conducted in Finland, the mortality rate was increased by 39 -fold in elderly patients undergoing an appendectomy [9]. Knowing that diagnosis may be delayed in elderly patients, the effectiveness of the scoring systems gains importance to avoid increased morbidity and mortality rates.

In the current study, we aimed to investigate the effectiveness of the RIPASA scoring method in the diagnosis of acute appendicitis in elderly patients. 
TA B L E 1. The Raja Isteri Pengiran Anak Saleha Appendicitis Scoring System, 2010 [7].

\begin{tabular}{|lc|}
\hline Variable & Score \\
\hline Gender & \\
\hline Female gender & 0.5 \\
\hline Male gender & 1 \\
\hline Age (years) & \\
$\leq 40$ & 1 \\
\hline 40 & 0.5 \\
\hline
\end{tabular}

Symptoms

\begin{tabular}{lc} 
Right iliac fossa pain & 1 \\
\hline Pain migratory to the right iliac fossa & 0.5 \\
\hline Anorexia & 1 \\
\hline Nausea and vomiting & 1 \\
\hline Duration of symptoms (hours) & \\
$<48$ & 1 \\
$\geq 48$ & 0.5 \\
\hline
\end{tabular}

Signs

\begin{tabular}{|lc} 
Right iliac fossa tenderness & 1 \\
\hline Guarding & 2 \\
\hline Rebound tenderness & 1 \\
\hline Rovsing sign & 2 \\
\hline Temperature between 37 and $39^{\circ} \mathrm{C}$ & 1 \\
\hline Laboratory & \\
\hline Leukocytosis & 1 \\
\hline Negative urine analysis & 1 \\
\hline Foreign nationality & 1 \\
\hline Maximum score & $\mathbf{1 6 . 5}$ \\
\hline
\end{tabular}

\section{Methods}

This study was approved by the local ethics committee of the Harran University Faculty of Medicine, Turkey (Approval number: HRU/20.04.20). The records of 68 patients aged over 65 years diagnosed with AA and who had undergone an appendectomy in the same institution based in Urfa, Turkey (a Eurasian country) between February 2015 and February 2020 were analyzed retrospectively. The demographic data of patients, including age, gender, duration of the hospitalization, and histopathological reports of the appendectomy materials, were recorded. The RIPASA score of the patients was calculated based on the anamnesis, clinical history, physical examination, and laboratory results of the patients. Patients with no acute appendicitis as diagnosed by microscopy were excluded from the study.

According to the RIPASA scores, the patients were divided into three groups as the low-score group (scores 4 to 7), the intermediate-score group (7.5 to 11.5), and the high-score group ( $\geq 12)$. The microscopy-based diagnoses were classified as follows: the normal appendix, the catarrhal stage, and complicated (perforated appendicitis and gangrenous appendicitis) $[10,11]$. The groups were compared according to the microscopy-based diagnoses.

\section{Statistical analysis}

SPSS 18 (SPSS Inc., Chicago, IL, USA) program was used for statistical analysis. Numerical data were presented as mean \pm standard deviation. The one-Sample Kolmogorov Smirnov test was used to evaluate the distribution of numerical data. The independent sample $t$-Test was used when the distribution was normal, and the Mann Whitney U test was used for nonnormal distribution. A Chi-square test was used for making comparisons between groups. Values with a $P$-value $<0.05$ were considered to be statistically significant.

\section{Results}

The mean age of the patients included in the study was $71.65 \pm$ 6.4 years. Thirty-seven (54\%) of the patients were male, and $31(46 \%)$ were female. The mean duration of hospital stay of the patients was $3.9 \pm 2.3$ (1-12 days). Eight of the patients considered in this series developed a wound infection, and one developed an intra-abdominal abscess in the post-operative period.

According to the calculated RIPASA score, there were 12 patients in the low-score group, 44 patients in the intermediatescore group, and 12 patients in the high-score group. When we classified the patients based on the microscopy-based diagnoses, a normal appendix or reactive lymphoid hyperplasia (non-complicated) was detected in ten patients, catarrhal stage appendicitis (non-complicated) was detected in 38 patients, and complicated appendicitis (gangrenous, perforated) was detected in 20 patients (Table 2). Comparing the RIPASA score with the histopathological data, we detected a sensitivity of $86.2 \%$, and specificity of $40 \%$, a positive predictive value of $89.3 \%$, a negative predictive value of $33.3 \%$, and a diagnostic accuracy rate of $79.4 \%$ in diagnosing AA (Table 3 ). Wound infection developed in eight patients, and intraabdominal abscess developed in one patient in the post-operative period. The average RIPASA value of these patients was 10.44 (8-14.5). Histopathological examination of nine patients who developed complications revealed gangrenous changes in two patients and perforation in four patients in addition to appendicitis.

A statistically significant correlation was found between the RIPASA scores of the patients and the pathological stages of their appendicitis $(P=0.00)$.

\section{Discussion}

Although acute appendicitis is a common problem, challenges in the diagnosis of AA still exist, especially in young or elderly patients and pregnant women [12]. A significant number of elderly patients are admitted to emergency services with complaints of abdominal pain [13]. Among these patients, acute appendicitis is the third most common cause of acute abdominal issues after intestinal obstruction and biliary diseases [14]. The complication rate increases in elderly patients since the presentations of appendicitis may be atypical in comparison to younger patients [3]. A study reported increased perforation rates in elderly patients when compared to younger patients 
TA B L E 2. Comparison of RIPASA scores obtained by microscopy-based diagnoses.

\begin{tabular}{|lcccc|}
\hline Microscopy Diagnosis & \multicolumn{3}{c}{ RIPASA score } & Total \\
\hline Normal & $\mathbf{1}$ & $\mathbf{2}$ & $\mathbf{3}$ & \\
& 4 & 6 & 0 & 10 \\
\hline Catarrhal stage & $40.0 \%$ & $60.0 \%$ & $0.0 \%$ & \\
& 8 & 30 & 0 & 38 \\
\hline Complicated & $21.1 \%$ & $78.9 \%$ & $0.0 \%$ & \\
\hline Perforated appendicitis, gangrenous appendicitis) & 0 & 8 & 12 & 20 \\
\hline & $0.0 \%$ & $40.0 \%$ & $60.0 \%$ & \\
\hline Total & 12 & 44 & 12 & 68 \\
\hline & $17.6 \%$ & $64.7 \%$ & $17.6 \%$ & \\
\hline
\end{tabular}

TA B L E 3. Diagnostic predictive values of the RIPASA score according to microscopy-based diagnosis.

\begin{tabular}{|lc|}
\hline Sensitivity & $86.2 \%$ \\
\hline Specificity & $40 \%$ \\
\hline Positive Predictive Value & $89.3 \%$ \\
\hline Negative Predictive Value & $33.3 \%$ \\
\hline Diagnostic Accuracy & $79.4 \%$ \\
\hline
\end{tabular}

due to the low rate of classic signs and symptoms and the delay in admission [15]. Perforation rates are between 20$30 \%$ in the general population; however, this rate rises to $50-70 \%$ in elderly people [16]. In our study, gangrenous or perforated (complicated) appendicitis was observed in 20 (29.4\%) patients. The perforation rates are related to the pre-hospital and intra-hospital delay that is mainly associated with the quality of care and the lower perforation rates in our study when compared with the aforementioned report. This may be caused by the easy access to health-care facilities and health-care professionals due to the advanced healthcaresystem planning in our country.

Acute appendicitis is diagnosed based on the history, detailed physical examination, and laboratory parameters. However, previous studies have reported that negative appendectomy and complicated appendectomy rates are particularly higher in children and patients aged over 60 years [17]. Therefore, various scoring systems have been developed in order to increase the diagnostic accuracy and reduce the rates of negative appendectomy and complicated appendicitis reported for acute appendicitis. The most well-known of these scoring systems include the Alvarado Score, the modified Alvarado Score, the Appendix Inflammatory Response Score, the Ohmann Score, and the Lintula Score. However, since these scoring systems gave different results in different ethnic groups, new scoring systems have been designed.

The RIPASA score is one of the scoring systems developed in 2010 that was widely used later on. There are 14 parameters in the RIPASA score. These parameters include the clinical history, physical examination, and several laboratory parameters. The total score in this scoring system is between 3 and 16.5. Acute appendicitis risk is lower in those with a score of less than 7, while the risk is relatively higher in those with a score of 7.5 and above [7, 18]. The RIPASA score, used especially in the Asian population, is an inexpensive, easy-to-use, and highly reliable quantitative scoring system that enables accurate and early diagnosis of acute appendicitis while significantly reducing the rate of negative appendicitis [19]. In a study conducted by Malik et al., it was shown that patients who were treated by surgical resection for acute appendicitis in a western population with a RIPASA score over 7.5 showed a sensitivity, specificity, positive predictive value rate, negative predictive value rate, and diagnostic accuracy of $85.39 \%, 69.86 \%, 84.06 \%, 72.86 \%$, and $80 \%$, respectively [20]. In a study conducted by Karapolat et al., it was shown that the RIPASA score obtained using simple clinical and laboratory data can be used to accurately and quickly diagnose acute appendicitis along with its possible pathological stage without the need for computed tomography [19]. In our study, when the RIPASA score in the elderly was compared with the histopathological data, its sensitivity was $86.2 \%$, its specificity was $40 \%$, while its positive predictive value, negative predictive value, and diagnostic accuracy rate were detected to be $89.3 \%, 33.3 \%$, and $79.4 \%$, respectively.

The retrospective nature of the study can be considered as a limitation. In addition, the relatively small size of the study represents preliminary results and needs to be supported with further large-scale studies.

\section{Conclusions}

In conclusion, the RIPASA score can be used in elderly patients for diagnosis of AA, considering its high accuracy rate. In elderly patients with a high RIPASA score, there is an increased risk for a pathological outcome of complicated (perforated or gangrenous) appendicitis. Therefore, the increased risk of complications may lead clinicians to be alert for potential risks for the clinical outcomes of elderly patients.

\section{AUTHOR CONTRIBUTIONS}

Study concept and design, acquisition of data: Osman Bardakci; Analysis and interpretation of data: Osman Bardakci, Faik Tatli; Preparation of study tools, drafting of manuscript: 
Osman Bardakci, Faik Tatli; Critical revision: Osman Bardakci, Faik Tatli.

\section{ETHICS APPROVAL AND CONSENT TO PARTICIPATE}

This study was approved by the local ethics committee of the Harran University Faculty of Medicine, Turkey (Approval number: HRU/20.04.20).

\section{ACKNOWLEDGMENT}

All authors contributed to the designation, data analysis, and writing of the study. The authors would like to thank all the participants involved in the study.

\section{FUNDING}

This research received no specific grant from any funding agency in the public, commercial, or not-for-profit sectors.

\section{CONFLICT OF INTEREST}

The authors declare that there is no conflict of interest regarding the publication of this article.

\section{DATA AVAILABILITY}

The data used to support the findings of this study are available from the corresponding author upon request.

\section{REFERENCES}

[1] Karami MY, Niakan H, Zadebagheri N, Mardani P, Shayan Z, Deilami I. Which one is better? Comparison of the acute inflammatory response, Raja Isteri Pengiran Anak Saleha Appendicitis and alvarado scoring systems. Annals of Coloproctology. 2017; 33: 227-231.

[2] Addiss DG, Shaffer N, Fowler BS, Tauxe RV. The epidemiology of appendicitis and appendectomy in the United States. American Journal of Epidemiology. 1990; 132: 910-925.

[3] Buckius MT, McGrath B, Monk J, Grim R, Bell T, Ahuja V. Changing epidemiology of acute appendicitis in the United States: study period 1993-2008. Journal of Surgical Research. 2012; 175: 185-190.

[4] Sheu B, Chiu T, Chen J, Tung M, Chang M, Young Y. Risk factors associated with perforated appendicitis in elderly patients presenting with signs and symptoms of acute appendicitis. ANZ Journal of Surgery. 2007; 77: $662-666$.
[5] Dado G, Anania G, Baccarani U, Marcotti E, Donini A, Risaliti A, et al. Application of a clinical score for the diagnosis of acute appendicitis in childhood: a retrospective analysis of 197 patients. Journal of Pediatric Surgery. 2000; 35: 1320-1322.

[6] Siddiqui ZR, Khaliq T, Shah SA. A new simple scoring system for the diagnosis of acute appendicitis. Journal of Pakistan Medical Students. 2011; 1: 32-37.

[7] Chong CF, Adi MI, Thien A, Suyoi A, Mackie AJ, Tin AS, et al. Development of the RIPASA score: a new appendicitis scoring system for the diagnosis of acute appendicitis. Singapore Medical Journal. 2010; 51: 220-225.

[8] Butt MQ, Chatha SS, Ghumman AQ, Farooq M. RIPASA score: a new diagnostic score for diagnosis of acute appendicitis. Journal of the College of Physicians and Surgeons. 2016; 24: 894-897.

[9] Kotaluoto S, Ukkonen M, Pauniaho S, Helminen M, Sand J, Rantanen T. Mortality related to appendectomy; A population based analysis over two decades in Finland. World Journal of Surgery. 2017; 41: 64-69.

[10] Riber C, Tønnesen H, Aru A, Bjerregaard B. Observer variation in the assessment of the histopathologic diagnosis of acute appendicitis. Scandinavian Journal of Gastroenterology. 1999; 34: 46-49.

[11] Karapolat B. Value of ultrasonography and the Raja Isteri Pengiran Anak Saleha Appendicitis Score in the diagnosis of acute appendicitis. ANZ Journal of Surgery. 2020; 90: 525-528.

[12] Eales L. Appendicitis and mimicking conditions. Lancet. 1976; 1: 704.

[13] Fagbohun CF, Toy EC, Baker B. The evaluation of acute abdominal pain in the elderly patient. Primary care update for OB/GYNS. 1999; 6: 181185.

[14] Franz MG, Norman J, Fabri PJ. Increased morbidity of appendicitis with advancing age. American Surgeon. 1995; 61: 40-44.

[15] Alvarado A. A practical score for the early diagnosis of acute appendicitis. Annals of Emergency Medicine. 1986; 15: 557-564.

[16] Temple CL, Huchcroft SA, Temple WJ. The natural history of appendicitis in adults. A prospective study. Annals of Surgery. 1995; 221: 278-281.

[17] Flum DR, Koepsell T. The clinical and economic correlates of misdiagnosed appendicitis: nationwide analysis. Archives of Surgery. 2002; 137: 799-804.

[18] Nongmaithem M, Rathod S, Ali I, Bawa AS, Singh G, Mishra S. Evaluation of Raja Isteri Pengiran Anak Saleha Appendicitis Score: a new appendicitis scoring system. Medical Journal of Dr. D. Y. Patil University. 2015; 8: 744-749.

[19] Karapolat B. Can RIPASA scoring system predict the pathological stage of acute appendicitis? Emergency Medicine International. 2019; 2019: 8140839.

[20] Malik MU, Connelly TM, Awan F, Pretorius F, Fiuza-Castineira C, El Faedy $\mathrm{O}$, et al. The RIPASA score is sensitive and specific for the diagnosis of acute appendicitis in a western population. International Journal of Colorectal Disease. 2017; 32: 491-497.

How to cite this article: Osman Bardakci, Faik Tatli. The effectiveness of the Raja Isteri Pengiran Anak Saleha Appendicitis Score in the diagnosis of acute appendicitis in elderly patients. Signa Vitae. 2021;17(3):214-217. doi:10.22514/sv.2021.027. 